\title{
Fluorescence imaging of membrane dynamics in living cells
}

\author{
Petra Weber \\ Michael Wagner \\ Herbert Schneckenburger \\ Hochschule Aalen \\ Institut für Angewandte Forschung \\ Beethovenstrasse 1 \\ D-73430 Aalen, Germany
}

\begin{abstract}
Methods of wide-field fluorescence microscopy for measuring membrane dynamics of living cells are described, including spectral imaging as well as anisotropy imaging of the membrane marker 6-dodecanoyl-2-dimethylamino naphthalene (laurdan). Plasma membranes are selected by illumination with an evanescent electromagnetic field and distinguished from intracellular membranes assessed by whole-cell illumination. While fluorescence spectra of laurdan appeared red-shifted with decreasing membrane stiffness, fluorescence anisotropy and rotational correlation times were reduced with increasing membrane fluidity. Membrane stiffness was found to increase with decreasing temperature and increasing amounts of cholesterol and was always higher for the plasma membrane than for intracellular membranes. These effects may have some clinical relevance in the research of drug resistance or cell aging. (๑ 2010 Society of Photo-Optical Instrumentation Engineers. [DOI: 10.1117/1.3470446]
\end{abstract}

Keywords: living cells; membranes; fluorescence microscopy; fluorescence anisotropy; total internal reflection; cholesterol; laurdan.

Paper 10099PR received Feb. 26, 2010; revised manuscript received Jun. 7, 2010; accepted for publication Jun. 9, 2010; published online Jul. 29, 2010.

\section{Introduction}

Membrane dynamics-including mechanical stiffness and exchange of lipid molecules (membrane fluidity) — has a large impact on cell function and may be an important factor for pathogenesis of, e.g., cancer ${ }^{1}$ or Alzheimer's disease. ${ }^{2}$ An essential component of lipid bilayers is cholesterol, which determines its fluidity and rigidity. Membrane dynamics has so far been deduced from measurements of fluorescence polarization $^{3}$ or fluorescence recovery after photobleaching $(\text { FRAP })^{4}$ using specific membrane markers. In addition, polarity-sensitive probes, e.g., 6-dodecanoyl-2-dimethylamino naphthalene (laurdan), whose electronic excitation energy is different in polar and nonpolar environments, ${ }^{5,6}$ proved to be useful for membrane studies. Once incorporated into cell membranes, the fluorescence spectrum of laurdan shows a shift toward longer wavelengths when the laurdan molecules come into contact with adjacent water molecules of the cytoplasm, e.g., when membrane stiffness decreases or when a phase transition from the tightly packed gel phase to the liquid crystalline phase of membrane lipids occurs. Fluorescence maxima around $440 \mathrm{~nm}$ and $490 \mathrm{~nm}$ have been observed, so far, and the generalized polarization

$$
\mathrm{GP}=\left(I_{440}-I_{490}\right) /\left(I_{440}+I_{490}\right),
$$

has been defined as a parameter of membrane stiffness, ${ }^{7}$ with $I_{440}$ corresponding to the fluorescence intensity at $440 \mathrm{~nm}$ and $I_{490}$ to that at $490 \mathrm{~nm}$.

Address all correspondence to Herbert Schneckenburger, Hochschule Aalen Institut für Angewandte Forschung, Beethovenstrasse 1, D-73430 Aalen, Germany. Tel: 49-7361-576-3401; Fax: 49-7361-576-3318; E-mail: herbert.schneckenburger@htw-aalen.de
In addition to fluorescence spectra, measurements of fluorescence anisotropy $r$ can give valuable information about membrane dynamics according to

$$
r=\left(I_{\|}-I_{\perp}\right) /\left(I_{\|}+2 I_{\perp}\right),
$$

with $I_{\|}$and $I_{\perp}$ corresponding to the fluorescence intensities with an electrical field vector parallel and perpendicular to the plane of incidence. The faster a molecule rotates between optical excitation and fluorescence, the lower is the expected value $r$. This value can be determined from steady state as well as from time-resolved fluorescence measurements. In the latter case, $r(t)$ decays exponentially after short laser pulse excitation according to

$$
r(t)=\left(r_{0}-r_{\infty}\right) e^{-t / \tau r}+r_{\infty},
$$

with the initial value $r_{0}$, the limiting value $r_{\infty}$ (observed at times that are long in comparison with the fluorescence lifetime and expressing that the rotational motion of a fluorophore may be hindered in one direction), and the rotational correlation time $\tau_{r}$ which is a direct measure of membrane viscosity. $^{3}$

While conventional wide-field microscopy permits simultaneous measurements of different cell membranesincluding the plasma membrane and various intracellular membranes - the plasma membrane can be examined selectively upon total internal reflection (TIR) of a laser beam ${ }^{8,9}$ propagating through a medium of refractive index $n_{1}$ (e.g., glass substrate) and meeting a second medium of refractive index $n_{2}<n_{1}$ (e.g., cell). Despite being totally reflected at all angles of incidence $\Theta$ that are greater than a critical angle

1083-3668/2010/15(4)/046017/5/\$25.00 @ 2010 SPIE 
$\Theta_{c}=\arcsin \left(n_{2} / n_{1}\right)$, the incident beam establishes an evanescent electromagnetic field that penetrates into the second medium and decays exponentially with the distance $z$ from the interface. According to the relation

$$
d=(\lambda / 4 \pi)\left(n_{1}^{2} \sin ^{2} \Theta-n_{2}^{2}\right)^{-1 / 2},
$$

penetration depths $d$ between about $60 \mathrm{~nm}$ and more than $300 \mathrm{~nm}$ are attained depending on the wavelength $\lambda$ and the angle of incidence $\Theta$.

The present paper reports on fluorescence microscopy of human U373-MG glioblastoma cells incubated with laurdan and presents spectral images and fluorescence anisotropy images upon variation of temperature as well as of the intracellular amount of cholesterol. Changes of cholesterol in cell membranes may affect protein interactions, ${ }^{10}$ e.g., in pathogenesis of Alzheimer's disease, ${ }^{11,12}$ and may have some influence on drug resistance ${ }^{13}$ as well as on cell aging. ${ }^{14}$ Plasma membranes illuminated selectively by TIR illumination are distinguished from intracellular membranes assessed by epiillumination of whole cells.

\section{Materials and Methods}

\subsection{Materials}

U373-MG human glioblastoma cells were obtained from the European Collection of Cell Cultures (ECACC No. 89081403). Cells were routinely grown in RPMI 1640 medium supplemented with $10 \%$ fetal calf serum and antibiotics at $37{ }^{\circ} \mathrm{C}$ and $5 \% \mathrm{CO}_{2}$. The hydrophobic membrane marker 6-dodecanoyl-2-dimethylamino naphthalene (laurdan) was obtained from Molecular Probes (Göttingen, Germany) and prepared as a $2 \mathrm{mM}$ Stock solution in ethanol. Water-soluble cholesterol and methyl- $\beta$-cyclodextrine $(\mathrm{M} \beta \mathrm{CD})$ were obtained from Sigma (Munich, Germany). After seeding of 150 cells $/ \mathrm{mm}^{2}$, cells were grown on microscope object slides for $48 \mathrm{~h}$ prior to rinsing with Earl's balanced salt solution (EBSS) and incubation with laurdan $(8 \mu \mathrm{M})$ or coincubation with laurdan $(8 \mu \mathrm{M})$ and $\mathrm{M} \beta \mathrm{CD}(4 \mathrm{mM})$ or coincubation with laurdan $(8 \mu \mathrm{M})$ and a $\mathrm{M} \beta \mathrm{CD}$ :cholesterol complex $(0.63 \mathrm{mM}: 0.1 \mathrm{mM})$ diluted in culture medium without serum. Cholesterol depletion after application of $\mathrm{M} \beta \mathrm{CD}$ as well as cholesterol enrichment after application of a $\mathrm{M} \beta \mathrm{CD}$ :cholesterol complex are well documented in the literature. ${ }^{15}$ After incubation for $60 \mathrm{~min}$ in each case, the intracellular amount of cholesterol was either reduced to about $50 \% \quad(\mathrm{M} \beta \mathrm{CD})$ or enriched to about $200 \%$ ( $\mathrm{M} \beta \mathrm{CD}$ :cholesterol complex), as determined with an Amplex Red Cholesterol Assay Kit (Molecular Probes) and visualized by coupling of cholesterol to the antibiotic filipin (Sigma). After rinsing with EBSS, cells were measured in an open aluminum chamber at variable temperatures ranging from $16{ }^{\circ} \mathrm{C}$ to $40{ }^{\circ} \mathrm{C}$ using a $63 \times / 0.90$ water immersion objective lens. This custom-made chamber (filled with a layer of 2 to $3 \mathrm{~mm}$ EBSS) contained a Peltier element for heating or cooling, as well as a calibrated thermocouple for temperature measurements in close vicinity to the measured part of the samples.

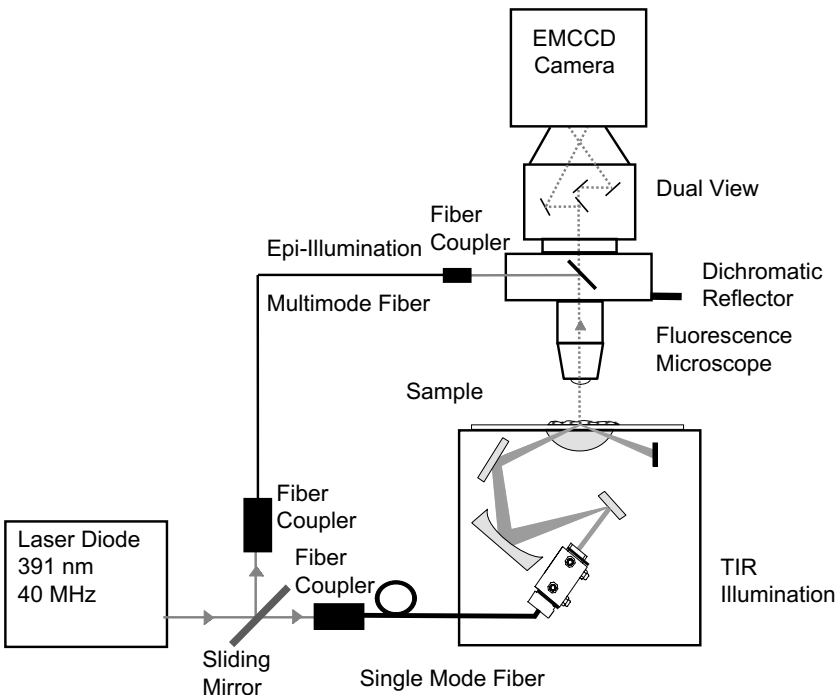

Fig. 1 Fluorescence microscope for spectral imaging or anisotropy imaging on TIR or epi-illumination. In some cases, the EM-CCD camera was replaced by a polychromator with an image-intensified detector or a time-gated image-intensifying camera.

\subsection{Experimental Setup}

As depicted in Fig. 1, a diode laser with high repetition pulses (LDH 400 with driver PDL 800-B, Picoquant, Berlin, Germany; wavelength: $391 \mathrm{~nm}$; pulse energy: $12 \mathrm{pJ}$; pulse duration: $55 \mathrm{ps}$; repetition rate: $40 \mathrm{MHz}$; average power: $0.5 \mathrm{~mW}$ ) was adapted to a fluorescence microscope (Axioplan 1, Carl Zeiss, Jena, Germany) using either a multimode fiber for epi-illumination of whole cells or a single-mode fiber-optic system (kineFlex-p-3-S-395, Point Source, Southampton, UK) together with a custom-made condenser unit $^{16}$ for illumination of the plasma membrane and adjacent cellular sites under total internal reflection (TIR). Commonly, an angle of incidence $\Theta=67 \pm 0.5 \mathrm{deg}$ was used, permitting a penetration depth of the evanescent wave of about $120 \pm 20 \mathrm{~nm}$, if refractive indices $n_{1}=1.515$ (glass substrate) and $n_{2}=1.37$ (cytoplasm) are assumed. For anisotropy measurements, the electrical field vector was always polarized perpendicular to the plane of incidence. Fluorescence images were recorded by an electron multiplying (EM)-CCD camera with Peltier cooling and a sensitivity below $10^{-16} \mathrm{~W} /$ pixel (DV887DC, ANDOR Technology, Belfast, UK $)^{17}$ in combination with appropriate spectral filters (bandpass filters for $454 \pm 20 \mathrm{~nm}$ and $497 \pm 20 \mathrm{~nm}$ used sequentially for spectral imaging or long-pass filter for $\lambda \geqslant 420 \mathrm{~nm}$ used for anisotropy imaging). For anisotropy imaging, two polarized fluorescence images (with an electrical field vector parallel and perpendicular to the plane of incidence) were generated by a polarization beamsplitter and detected simultaneously on different segments of the EM-CCD camera (Dual View, Optical Insights, Tucson, Arizona). Slight differences of sensitivity within the two detection paths were corrected by determination of the so-called $\mathrm{G}$ factor, i.e., the factor of fluorescence intensities measured within the two paths when in both cases the polarization of emission and excitation light was parallel. For additional measurements of fluorescence spectra, a custom-made polychromator together with an image- 




Fig. 2 Fluorescence spectra of single U373-MG glioblastoma cells incubated with laurdan $(8 \mu \mathrm{M}, 1 \mathrm{~h})$ at a natural amount of cholesterol (lower curve) or on cholesterol enrichment (to 200\%; middle curve) or cholesterol depletion (to $50 \%$, upper curve) at $T=24{ }^{\circ} \mathrm{C}$ (whole cell illumination at $\lambda=391 \mathrm{~nm}$ ); molecular structure of laurdan.

intensifying detection unit (IMD 4562, Hamamatsu Photonics, Ichino-Cho, Japan) was fixed on top of the microscope, ${ }^{18}$ whereas for measurements of the rotational correlation time, fluorescence decay kinetics was recorded with a time-gated image-intensifying camera (Picostar HR 12; LaVision, Göttingen, Germany) at a temporal resolution of 200 ps (Ref. 19).

\section{Results}

\subsection{Spectral Imaging}

The fluorescence spectrum of U373-MG glioblastoma cells incubated with laurdan is depicted in Fig. 2 together with the molecular structure of laurdan. All spectra are related to single cells either without further treatment (lower curve) or on enrichment of cholesterol (to about $200 \%$ of its natural amount; middle curve) or on cholesterol depletion (to about $50 \%$ of its natural amount; upper curve). While absolute fluorescence intensities varied from cell to cell up to a factor of about 2 , the intensity ratio of the fluorescence bands around $440 \mathrm{~nm}$ and $490 \mathrm{~nm}$ increased with the amount of cholesterol. This finding can be described by an increase of the generalized polarization (GP) with increasing membrane stiffness according to Eq. (1).

GP values determined from fluorescence images recorded within both spectral bands are depicted in Fig. 3 as a function of the intracellular amount of cholesterol on illumination of whole cells [(a): epi-illumination] and plasma membranes [(c): TIR illumination]. Corresponding images of fluorescence intensity [(b), (d), recorded at $\lambda \geqslant 420 \mathrm{~nm}]$ as well as a color scale for the GP values have been added. This figure proves the increasing GP values with increasing amounts of cholesterol and shows that GPs are always higher for the plasma membranes than for inner parts of the cells. This holds for all temperatures in the range $16{ }^{\circ} \mathrm{C} \leqslant \mathrm{T} \leqslant 40{ }^{\circ} \mathrm{C}$, although GP decreases continuously with increasing temperature (data not shown).

\subsection{Fluorescence Anisotropy Imaging}

Polarized measurements of laurdan fluorescence in U373-MG glioblastoma cells always showed higher fluorescence intensity within the plane of the incident electrical field vector than perpendicular to this plane. In addition, time-resolved mea- (a)

(b)

(c)

(d)

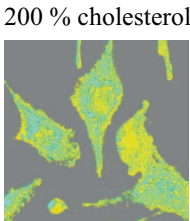

$100 \%$ cholesterol
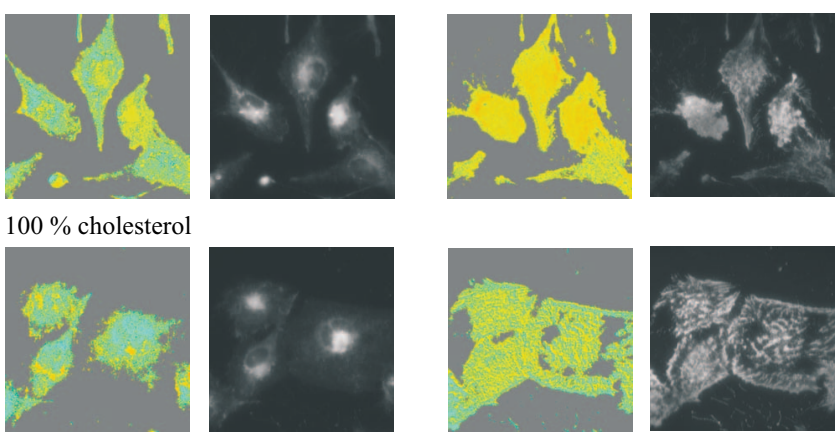

$50 \%$ cholesterol
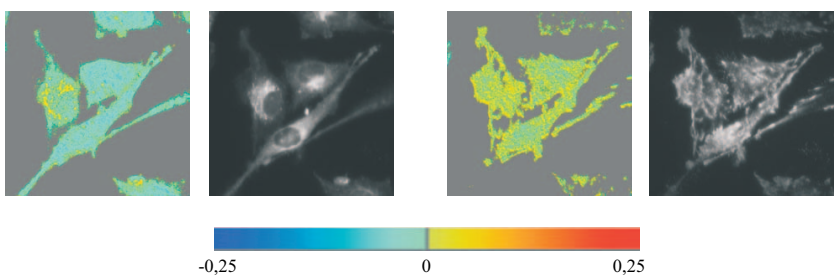

0,25

Fig. 3 GP images [(a), (c); calculated according to Eq. (1)] and corresponding fluorescence intensities [(b), (d); recorded at $\lambda \geqslant 420 \mathrm{~nm}]$ of U373-MG glioblastoma cells incubated with laurdan as a function of intracellular cholesterol amount for whole cells [(a), (b): epiillumination] and plasma membranes [(c), (d): TIR illumination] at $T$ $=24{ }^{\circ} \mathrm{C}$ (image size: $140 \mu \mathrm{m} \times 140 \mu \mathrm{m}$ each); scale of GP values.

surements after picosecond laser pulse excitation showed a fluorescence decrease with a lifetime around 5 ns (Ref. 19) for both polarizations as well as some delayed increase for the polarization perpendicular to the plane of incidence.

Images of steady state anisotropy determined according to Eq. (2) are depicted in Fig. 4 for whole-cell illumination and in Fig. 5 for TIR illumination as a function of temperature and intracellular amount of cholesterol. These figures clearly show increasing anisotropies with increasing amounts of cholesterol and decreasing temperatures. In addition, anisotropy values are always higher for the plasma membranes (visualized as cell edges on epi-illumination and cell surfaces on TIR illumination) than for inner parts of the cells including the nuclear membrane. A similar result is obtained if instead of the fluorescence anisotropy $r$, the rotational correlation time $\tau_{r}$ is determined from fluorescence decay kinetics, as depicted in Fig. 6. Again, $\tau_{r}$ decreases with temperature and increases with the amount of cholesterol (not shown) due to decreasing membrane viscosity in the first case and increasing viscosity in the second case. Obviously, the most pronounced change of $\tau_{r}$ occurs in the temperature range between $24{ }^{\circ} \mathrm{C}$ and $32^{\circ} \mathrm{C}$.

\section{Discussion}

Several parameters characterizing membrane dynamics of living cells have been reported. While the generalized polarization (GP) of laurdan characterizes mechanical stiffness, the fluorescence anisotropy $r$ and the rotational correlation time $\tau_{r}$ seem to characterize membrane viscosity or (inverse to viscosity) membrane fluidity. Obviously, similar information can be deduced from $r$ and $\tau_{r}$. GP on one hand and $r$ as well as $\tau_{r}$ on the other hand show a pronounced decrease with 


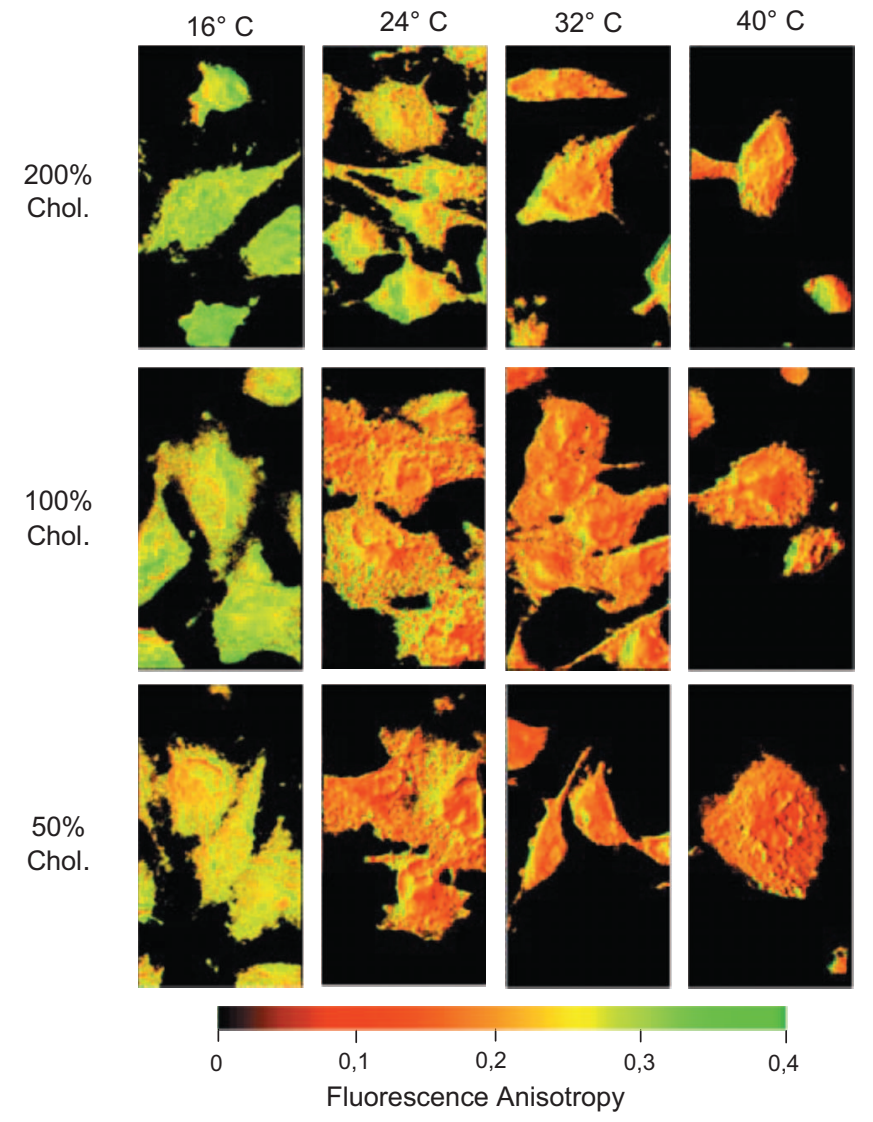

Fig. 4 Fluorescence anisotropy images of U373-MG glioblastoma cells incubated with laurdan as a function of temperature and intracellular cholesterol amount on whole-cell illumination (image size: $70 \mu \mathrm{m} \times 140 \mu \mathrm{m}$ each).

temperature and an increase with the intracellular amount of cholesterol. This proves an inverse behavior of mechanical stiffness and fluidity of cell membranes. Experiments of staining cholesterol with fluorescent filipin showed that on cholesterol depletion with methyl- $\beta$-cyclodextrine $(\mathrm{M} \beta \mathrm{CD})$, this depletion occurred primarily from inner parts of the cells, whereas comparably high cholesterol amounts were maintained in the plasma membranes. ${ }^{20}$ This explains their higher stiffness and lower fluidity in comparison with intracellular membranes.

A decrease of membrane stiffness with increasing temperature has been well documented for artificial membranes, ${ }^{5-7}$ but only in a few cases for living cells. ${ }^{21}$ While in artificial membranes, this decrease occurred in a small temperature interval (typically, around $40{ }^{\circ} \mathrm{C}$ ), it occurred more gradually in a temperature range between about $15^{\circ} \mathrm{C}$ and $40{ }^{\circ} \mathrm{C}$ for living cells. This is in good agreement with our own experiments and may be explained by the fact that several different membranes are expected to interfere.

It is of long-term interest to know how cholesterol may affect intermolecular interactions in cell membranes and whether it might favor or prevent pathogenesis of pathological processes. Just recently, we could show that enrichment as well as depletion of cholesterol could prevent interaction of amyloid precursor protein (APP) and $\beta$-secretase, ${ }^{12}$ which favors the production of amyloid peptide in the pathogenesis of

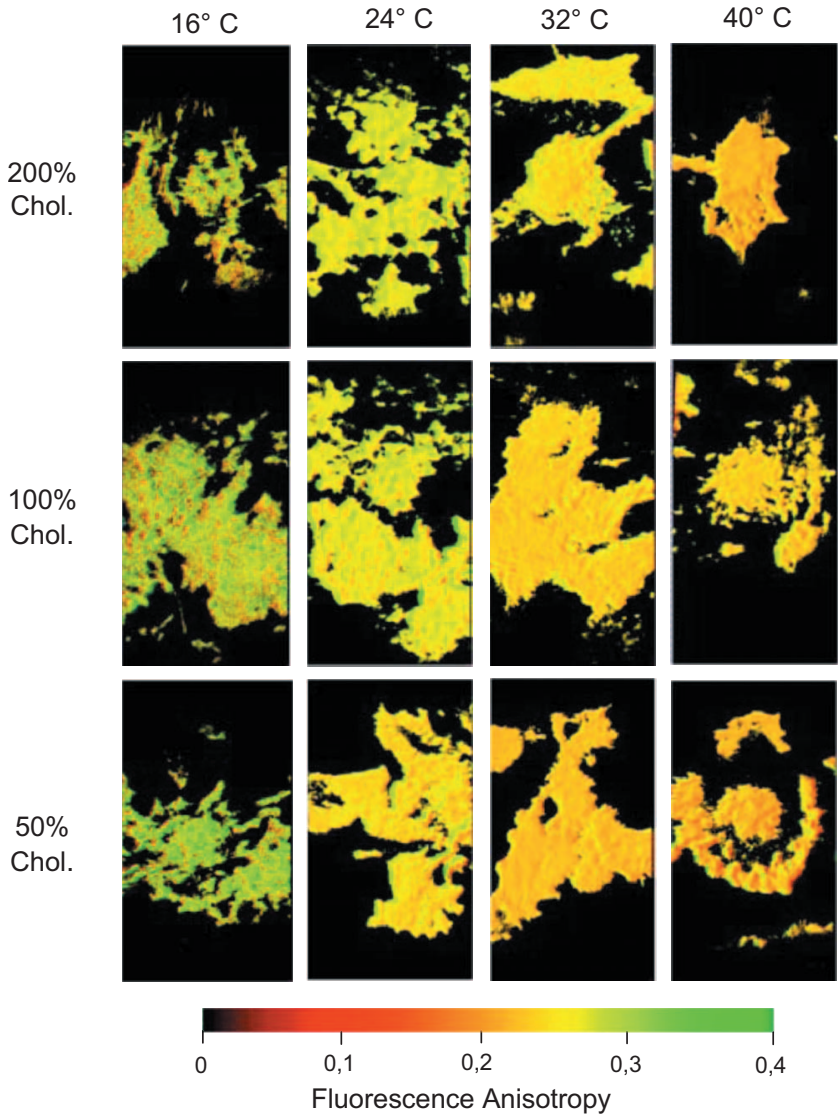

Fig. 5 Fluorescence anisotropy images of U373-MG glioblastoma cells incubated with laurdan as a function of temperature and intracellular cholesterol amount on TIR illumination (image size: $70 \mu \mathrm{m}$ $\times 140 \mu \mathrm{m}$ each).

Alzheimer's disease. In addition, an influence of cholesterol on cell aging is well described in the literature ${ }^{14}$ and verified by our own experiments (not shown): Aging cells of subcultures 38-42 (i.e., cells split 38-42 times) were stiffer than

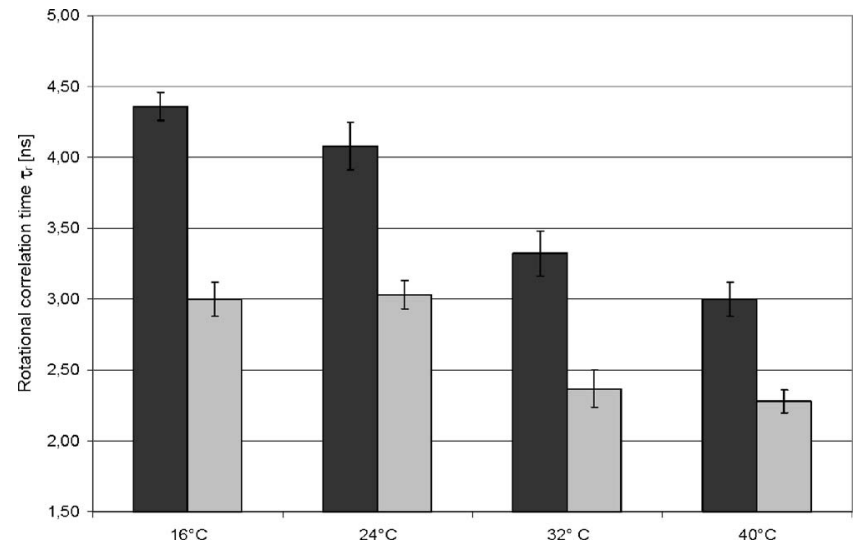

Fig. 6 Temperature dependence of the rotational correlation time of U373-MG glioblastoma cells incubated with laurdan on TIR (black bars: plasma membrane) and epi-illumination (gray bars: whole cell); median values \pm median absolute deviations (MADs) of 20 single-cell measurements in each case. 
cells of the subcultures $18-24$ generally used for our experiments.

While fluorescence spectra $^{5-7,21,22}$ and anisotropy ${ }^{22}$ measurements of laurdan have been well documented in the literature, only few imaging data of living cells are available so far. $^{23,24}$ Therefore, it was a main purpose of this paper to present spectral and anisotropy images of single cells and to compare laurdan fluorescence arising from plasma membranes with that arising from whole cells (and dominated by intracellular membranes). Switching of the illumination mode between total internal reflection (TIR) and epi-illumination is performed easily by two different illumination paths and a sliding mirror, as depicted in Fig. 1. TIR illumination with a hemispherical or hemicylindrical glass prism (Fig. 1) has further advantages in comparison with commercial TIR devices using high aperture objective lenses: easy variation of the penetration depth of the evanescent electromagnetic field by variation of the angle of incidence, and use of any kind of objective lens, even of rather low numerical aperture. This is essential for selection of larger object fields and for maintaining the polarization of light in fluorescence anisotropy measurements.

Imaging experiments profit to a large extent from the availability of highly sensitive EM-CCD cameras with excellent signal-to-noise ratio. Microscopic resolution in spectral and anisotropy imaging, however, is commonly limited by the Abbé criterion to about $200 \mathrm{~nm}$ but can be improved to a few nanometers in the axial direction by variable-angle TIR microscopy. ${ }^{16}$ So, microdomains of different membrane stiffness or fluidity can be visualized down to diameters around $200 \mathrm{~nm}$, whereas cholesterol-enriched lipid rafts of only a few nanometers in size cannot be resolved. ${ }^{25}$ The excellent axial resolution, however, permits us to measure cholesteroldependent cellular adhesions on a substrate in a nanometer scale, and loosening of those adhesions on depletion of cholesterol-finally leading to detachment of whole cellshas previously been well documented. ${ }^{26}$

\section{Acknowledgments}

The authors thank C. Hintze (Hochschule Aalen) for cell cultivation as well as P. Kruse and E. Winkler (ILM Ulm) for their support in quantitative measurements of cholesterol. Research was funded by the Bundesministerium für Bildung und Forschung (BMBF) and the Land Baden-Württemberg.

\section{References}

1. M. Sok, M. Senturc, M. Schara, J. Stare, and T. Rott, "Cell membrane fluidity and prognosis of lung cancer," Ann. Thorac. Surg. 73, 15671571 (2002).

2. N. B. Chauhan, "Membrane dynamics, cholesterol homeostasis, and Alzheimer's disease," J. Lipid Res. 44(11), 2019-2029 (2003).

3. J. R. Lakowicz, Principles of Fluorescence Spectroscopy, Plenum Press, New York and London (2006).

4. N. L. Thompson, A.-W. Drake, L. Chen, and W. V. Broek, "Equilibrium, kinetics, diffusion and self-association of proteins at membrane surfaces: measurement by total internal reflection fluorescence microscopy," Photochem. Photobiol. 65, 39-46 (1997).

5. T. Parasassi, G. de Stasio, A. d'Ubaldo, and E. Gratton, "Phase fluctuation in phospholipid membranes revealed by laurdan fluorescence," Biophys. J. 57, 1179-1186 (1990).

6. T. Parasassi, E. K. Krasnowska, L. Bagatolli, and E. Gratton, "Laur- dan and prodan as polarity-sensitive fluorescent membrane probes," J. Fluoresc. 6, 365-373 (1998).

7. T. Parasassi, G. de Stasio, G. Ravagnan, R. M. Rusch, and E. Gratton, "Quantitation of lipid phases in phospholipid vesicles by the generalized polarization of laurdan fluorescence," Biophys. J. 60, 179-189 (1991).

8. D. Axelrod, "Cell-substrate contacts illuminated by total internal reflection fluorescence," J. Cell Biol. 89, 141-145 (1981).

9. H. Schneckenburger, "Total internal reflection fluorescence microscopy: technical innovations and novel applications," Curr. Opin. Biotechnol. 16, 13-18 (2005).

10. K. Simons and R. Ehehalt, "Cholesterol, lipid rafts, and disease," $J$. Clin. Invest. 110, 597-603 (2002).

11. G. P. Eckert, N. J. Cairns, A. Maras, W. F. Gattaz, and W. E. Muller, "Cholesterol modulates the membrane-disordering effects of betaamloyid peptides in the hippocampus: specific changes in Alzheimer's disease," Dementia Geriatr. Cognit. Disord. 11, 181-186 (2000).

12. C. A. F. von Arnim, B. von Einem, P. Weber, M. Wagner, D. Schwanzar, R. Spoelgen, W. S. L. Strauss, and H. Schneckenburger, "Impact of cholesterol level upon APP and BACE proximity and APP cleavage," Biochem. Biophys. Res. Commun. 370, 207-212 (2008).

13. C. H. Storch, R. Eehehalt, E. Haefeli, and J. Weiss, "Localization of the human breast cancer resistance protein (BCRP/ABCG2) in lipid rafts/caveolae and modulation of its activity in vitro," J. Pharmacol. Exp. Ther. 323, 257-264 (2007).

14. T. Parasassi, M. di Stefano, G. Ravagnan, O. Sapora, and E. Gratton, "Membrane aging during cell growth ascertained by laurdan generalized polarization," Exp. Cell Res. 202, 432-439 (1992).

15. A. E. Christian, M. P. Haynes, M. C. Phillips, and G. H. Rothblat, "Use of cyclodextrins for manipulating cellular cholesterol content," J. Lipid Res. 38, 2264-2272 (1997).

16. K. Stock, R. Sailer, W. S. L. Strauss, M. Lyttek, R. Steiner, and H. Schneckenburger, "Variable-angle total internal reflection fluorescence microscopy (VA-TIRFM): realization and application of a compact illumination device," J. Microsc. 211, 19-29 (2003).

17. C. G. Coates, D. J. Denvir, N. G. McHale, K. D. Thornbury, and M. A. Hollywood, "Optimizing low-light microscopy with backilluminated electron multiplying charge-coupled device: enhanced sensitivity, speed, and resolution," J. Biomed. Opt. 9(6), 1244-1252 (2004).

18. H. Schneckenburger, M. H. Gschwend, R. Sailer, H.-P. Mock, and W. S. L. Strauss, "Time-gated fluorescence microscopy in molecular and cellular biology," Cell. Mol. Biol. (Paris) 44, 795-805 (1998).

19. H. Schneckenburger, M. Wagner, M. Kretzschmar, W. S. L. Strauss, and R. Sailer, "Laser-assisted fluorescence microscopy for measuring cell membrane dynamics," Photochem. Photobiol. Sci. 3, 817-822 (2004).

20. P. Weber, M. Wagner, W. S. L. Strauss, and H. Schneckenburger, "Fluorescence imaging of cholesterol and temperature dependent cell membrane dynamics," in Biophotonics 2007: Optics in Life Science, J. Popp and G. V. Bally, Eds., Proc. SPIE 6633, 663305 (2007).

21. C. F. Chapman, Y. Liu, G. J. Sonek, and B. Tromberg, "The use of exogenous fluorescent probes for temperature measurements in single living cells," Photochem. Photobiol. 62, 416-425 (1995).

22. F. M. Harris, K. B. Best, and J. D. Bell, "Use of laurdan fluorescence intensity and polarization to distinguish between changes in membrane fluidity and phospholipid order," Biochim. Biophys. Acta $\mathbf{1 5 6 5}$, 123-128 (2002).

23. W. Yu, P. T. C. So, T. French, and E. Gratton, "Fluorescence general polarization of cell membranes: a two-photon scanning microscopy approach," Biophys. J. 70, 626-636 (1996).

24. K. Gaus, S. Le Lay, N. Balasubramanian, and M. A. Schwartz, "Integrin mediated adhesion regulates membrane order," J. Cell Biol. 174, 725-734 (2006).

25. K. Gaus, T. Zech, and T. Harder, "Visualizing membrane microdomains by laurdan 2-photon microscopy," Mol. Membr Biol. 23 , 41-48 (2006).

26. M. Wagner, P. Weber, W. S. L. Strauss, H.-P. Lassalle, and H. Schneckenburger, "Nanotomography of cell surfaces with evanescent fields," Adv. Opt. Technol., article ID 254317 (2008). 